\title{
Pure neuritic leprosy presenting as ulnar nerve neuropathy: a case report of electrodiagnostic, radiographic, and histopathological findings
}

\author{
Russell Payne, MD, ${ }^{1}$ Jennifer Baccon, MD, PhD, ${ }^{2}$ John Dossett, MD, ${ }^{3}$ David Scollard, MD, PhD, ${ }^{5}$ \\ Debra Byler, MD, ${ }^{4}$ Akshal Patel, MD, ${ }^{1}$ and Kimberly Harbaugh, MD ${ }^{1}$
} Departments of ${ }^{1}$ Neurosurgery, ${ }^{2}$ Pathology, ${ }^{3}$ Pediatric Infectious Disease, and ${ }^{4}$ Neurology, Penn State Milton S. Hershey Medical
Center, Hershey, Pennsylvania; and ${ }^{5}$ National Hansen's Disease Programs Laboratory, Baton Rouge, Louisiana

\begin{abstract}
Hansen's disease, or leprosy, is a chronic infectious disease with many manifestations. Though still a major health concern and leading cause of peripheral neuropathy in the developing world, it is rare in the United States, with only about 150 cases reported each year. Nevertheless, it is imperative that neurosurgeons consider it in the differential diagnosis of neuropathy.
\end{abstract}

The causative organism is Mycobacterium leprae, which infects and damages Schwann cells in the peripheral nervous system, leading first to sensory and then to motor deficits. A rare presentation of Hansen's disease is pure neuritic leprosy. It is characterized by nerve involvement without the characteristic cutaneous stigmata. The authors of this report describe a case of pure neuritic leprosy presenting as ulnar nerve neuropathy with corresponding radiographic, electrodiagnostic, and histopathological data.

This 11-year-old, otherwise healthy male presented with progressive right-hand weakness and numbness with no cutaneous abnormalities. Physical examination and electrodiagnostic testing revealed findings consistent with a severe ulnar neuropathy at the elbow. Magnetic resonance imaging revealed diffuse thickening and enhancement of the ulnar nerve and narrowing at the cubital tunnel. The patient underwent ulnar nerve decompression with biopsy. Pathology revealed acid-fast organisms within the nerve, which was pathognomonic for Hansen's disease. He was started on antibiotic therapy, and on follow-up he had improved strength and sensation in the ulnar nerve distribution.

Pure neuritic leprosy, though rare in the United States, should be considered in the differential diagnosis of those presenting with peripheral neuropathy and a history of travel to leprosy-endemic areas. The long incubation period of $M$. leprae, the ability of leprosy to mimic other conditions, and the low sensitivity of serological tests make clinical, electrodiagnostic, and radiographic evaluation necessary for diagnosis. Prompt diagnosis and treatment is imperative to prevent permanent neurological injury.

http://thejns.org/doi/abs/10.3171/2014.9.JNS142210

KEY WORDS leprosy; neurolysis; decompression; entrapment; ulnar nerve; neuropathy; peripheral nerve

$\mathrm{H}$ ANSEN's disease, or leprosy, was first identified in patients in 1873 by Dr. Gerhard Armauer Hansen. It is a chronic infectious disease with a multitude of clinical manifestations. Though still a major health concern and leading cause of peripheral neuropathy in developing countries, it is rare in the United States, with only about 150 new cases reported each year..$^{30,31}$ The causative organism is Mycobacterium leprae, an acid-fast bacterium, which is thought to be spread by airborne droplets. $M$. leprae primarily infects Schwann cells in the periph- eral nervous system as well as histiocytes in the dermis. Manifestation of the disease depends on the host immune response and can be quite varied. The incubation period ranges from 3 months to 40 years, although the average is 7 years. ${ }^{22,30}$ Leprosy involves major nerves at and above locations surgeons commonly address for compressive neuropathy such as the ulnar nerve at the elbow, tibial nerve at the ankle, median nerve at the wrist, and peroneal nerve at the knee. ${ }^{9,21,22}$ In the pure neuritic form of leprosy, there are no significant cutaneous manifestations, making diagnosis 
particularly challenging. ${ }^{1,2}$ We describe the diagnosis and management of a case of pure neuritic leprosy manifesting as ulnar neuropathy at the elbow to demonstrate the potential diagnostic challenges in the modern imaging era and to discuss surgical considerations related to leprosy.

\section{Case Report}

\section{Presentation and Examination}

An 11-year-old, otherwise healthy male was referred for evaluation of a progressive right ulnar palsy and abnormal MRI. His symptoms began 9 months earlier with hand paresthesias followed by numbness. He also complained of a 5-month history of progressive hand weakness and pain involving his right medial elbow, forearm, wrist, and thumb. He had no history of neck or radicular pain, denied recent trauma or viral illness, and had no family history of neurological disease. He was delivered full-term by cesarean section in India and lived there until the age of 5 years, when he moved to the United States. He had spent 6 weeks in India visiting family several months prior to the onset of his symptoms. His medical history was otherwise unremarkable, and his immunizations were up to date.

Physical examination findings were limited to the right upper limb. The ulnar nerve was palpably enlarged in the medial arm. He had loss of pin sensation in the ulnar distribution of the hand as well as in the dorsum of the hand generally. Motor exam revealed severe ulnar palsy with minimal function of intrinsic hand muscles innervated by the ulnar nerve and significant weakness in the flexor digitorum profundus to the fourth and fifth digits with associated clawing. Median and radial motor function was normal.

\section{Imaging Studies}

On MRI studies, the ulnar nerve was thickened diffusely from the midhumeral level into the forearm with a maximal diameter of $12 \mathrm{~mm}$ (Fig. 1). It was isointense to muscle on T1 and hyperintense on T2 and STIR sequences. The nerve demonstrated intense contrast enhancement (Fig. 2). There was a questionable additional lesion involving the median nerve versus an enhancing lymph node just proximal to the elbow.

\section{Electrodiagnostic Studies}

Nerve conduction studies revealed an absent right ulnar sensory potential and motor potential at the first dorsal interosseous muscle. The ulnar motor potential at the abductor digit minimi muscle was significantly reduced in amplitude at $0.2 \mathrm{mV}$ (left $5.5 \mathrm{mV}$ ). The radial sensory potential was also significantly reduced at $2.5 \mu \mathrm{V}$ (left $75.7 \mu \mathrm{V})$. Left-sided responses, right median responses, and right radial motor responses were normal in duration and amplitude. Electromyography studies revealed significant abnormal spontaneous activity and no voluntary motor unit activation in the ulnar-innervated forearm and intrinsic musculature of the hand; otherwise, the studies were normal.

\section{Preoperative Evaluation and Operation}

Because the differential included leprosy, the patient
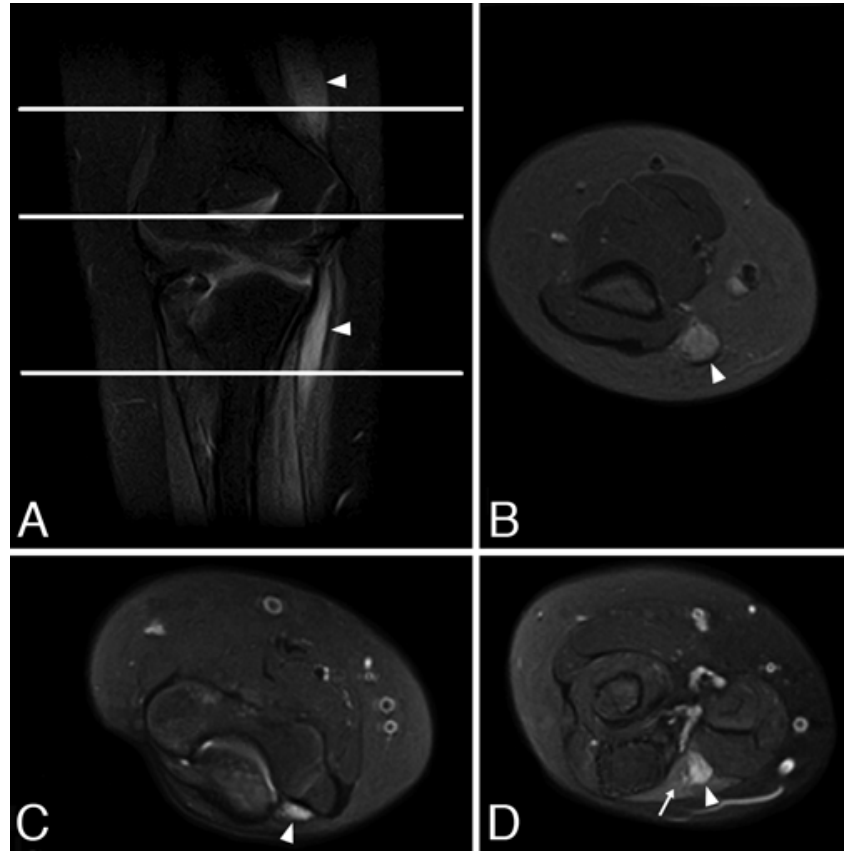

FIG. 1. T2-weighted fat saturation images showing the hyperintense ulnar nerve (arrowheads) and its course in the arm. A: The ulnar nerve is enlarged proximal to elbow. B: Within the cubital tunnel, the nerve decreases substantially in size. C: Just distal to the cubital tunnel, the nerve again becomes thickened. D: Increased signal consistent with denervation myositis (arrow) can also be seen in the flexor carpi ulnaris muscle at this level.

was referred for a pediatric infectious disease consultation. The National Hansen's Disease (Leprosy) Clinical Center was also contacted. As the patient had no clear cutaneous manifestation amenable to slit-skin biopsy and given the relative constriction of the ulnar nerve at the elbow in the face of a severe ulnar palsy, surgical exploration was recommended. At surgery, the ulnar nerve above the elbow was enlarged and firm. A relative constriction at the cubital tunnel was identified and released. Using electrophysiological monitoring and microscopic dissection, we removed a small nonfunctioning fascicle and sent it for pathological evaluation.

\section{Pathological Evaluation}

Sections stained with $\mathrm{H} \& \mathrm{E}$ revealed severe granulomatous and lymphocytic infiltration with little residual nerve visualized (Fig. 3). Fite stains performed at the national Hansen's disease laboratory revealed moderate acid-fast organisms within the specimen whose frequency led to the patient's classification in the multibacillary, midborderline to borderline lepromatous (BB-BL) portion of the disease spectrum (Fig. 4).

\section{Postoperative Course}

After rendering a diagnosis, we started the patient on a 3-drug regimen including rifampin, clofazimine, and dapsone with a planned duration of 2 years. He was also given a 3-month course of steroids. At 8 months postoperatively, the radial distribution numbness had resolved and the ulnar distribution numbness was improving. The clawing had resolved, and the flexor digitorum profundus 


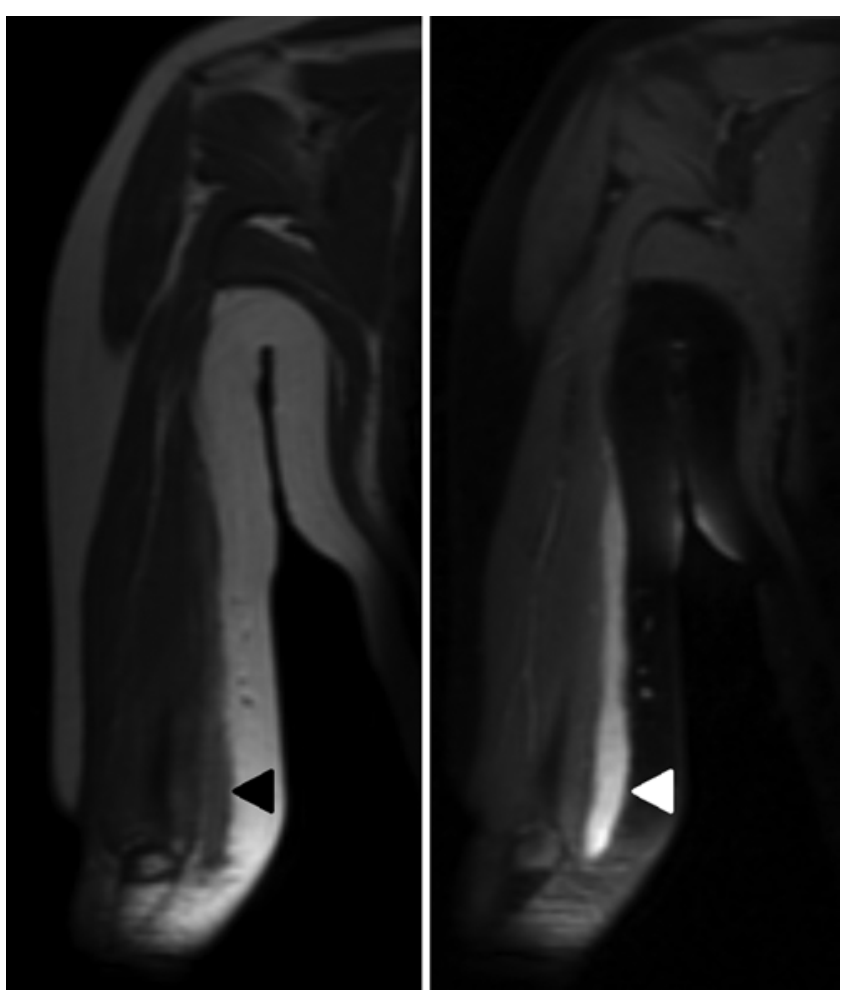

FIG. 2. Precontrast (left) and postcontrast (right) coronal T1-weighted MR images of the right arm. The thickened ulnar nerve (arrowheads) is readily visible in the postcontrast scan.

weakness had improved, but the severe ulnar interosseous muscle weakness persisted.

\section{Discussion \\ General Comments}

Leprosy remains a major cause of neurological morbidity worldwide, ${ }^{13,16,22,23}$ and with a mobile population traveling to and from endemic regions, the disease needs to be considered in the differential diagnosis of neuropathy. Typically, the diagnosis is made by taking a history and completing a physical exam and slit-skin smear or skin biopsy. In a nonendemic region, travel history, rash, and anesthetic skin may not be obvious unless actively sought. In the Americas, including the southern United States, in- teraction with the wild armadillo, the only other natural host of M. leprae, may result in transmission of the disease, ${ }^{28,31}$ so a travel history within the United States is also valuable.

While only 150 new cases are diagnosed in the United States each year, in 2009 it was estimated that more than 7000 people previously registered with the National Hansen's Disease Program might still be living in the United States. Because leprosy can be difficult to eradicate even with current multidrug therapy, leprosy patients may present with disease years after the initial diagnosis. ${ }^{17,32}$

\section{Diagnosis and Imaging}

When presented in this case report format, the diagnosis of leprosy seems obvious; however, the pure neuritic form of the disease presenting in a nonendemic area complicated the diagnosis. The patient had emigrated from an endemic region, but his father, who is a physician, noted that the patient had had no known contact with an infected individual. As $95 \%$ of individuals have a natural immunity to the microorganism, concern for leprosy was minimalized. ${ }^{1}$ Electrodiagnostic studies were helpful in delineating the extent of nerve involvement but were not specific to any particular diagnosis. The imaging studies were interpreted as being consistent with perineurioma, and neurological specialists at a nationally recognized institution counseled the family against surgical intervention. This is in keeping with recommendations in recent literature against biopsy of perineuriomas given their classic MRI appearance. ${ }^{20,24}$ While the sciatic nerve is the most common site of perineurioma involvement, ulnar nerve involvement has been reported. ${ }^{19}$

In addition to perineurioma, the differential diagnosis of nerve thickening, increased T2 signal, and contrast enhancement on MRI studies is broad and includes neoplastic entities such as nerve sheath tumors and lymphoma, inherited diseases, infectious entities such as leprosy, and inflammatory processes such as inflammatory pseudotumor of nerve. ${ }^{4,5,18,36}$ In our experience, the T2 signal change and enhancement in our case were more intense than typically seen in perineuriomas and more closely mimicked the T2 signal of a nerve sheath tumor. It is important to note both that the MRI appearance of lepromatous nerves can vary depending on the severity of the neuritis and that the resolution of changes may cor-

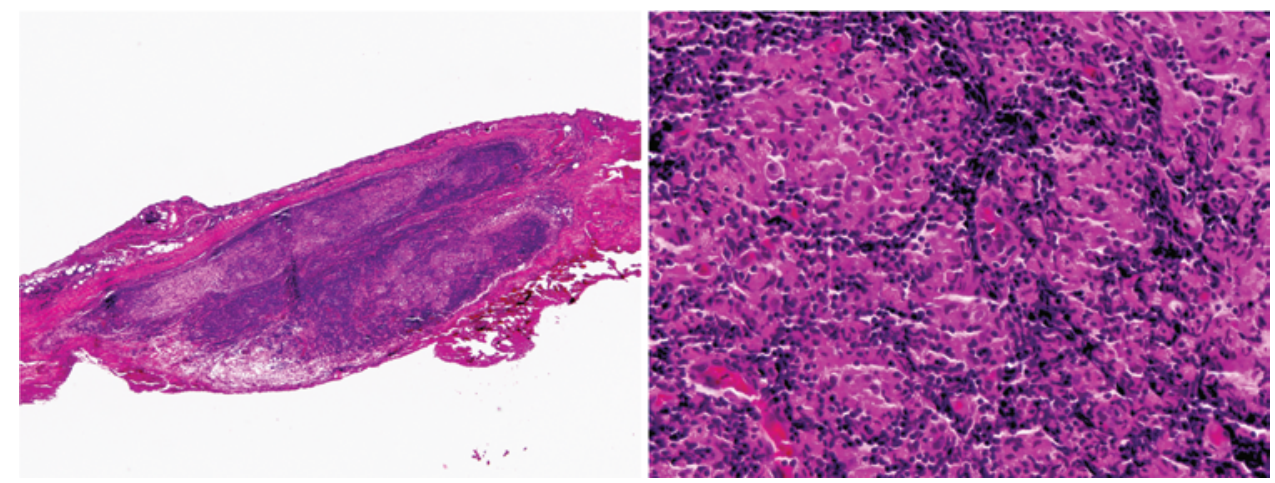

FIG. 3. Nerve biopsy showing lymphocytic and granulomatous inflammation. $H$ \& E, original magnification $\times 40$ (left) and $\times 400$ (right). Figure is available in color online only. 

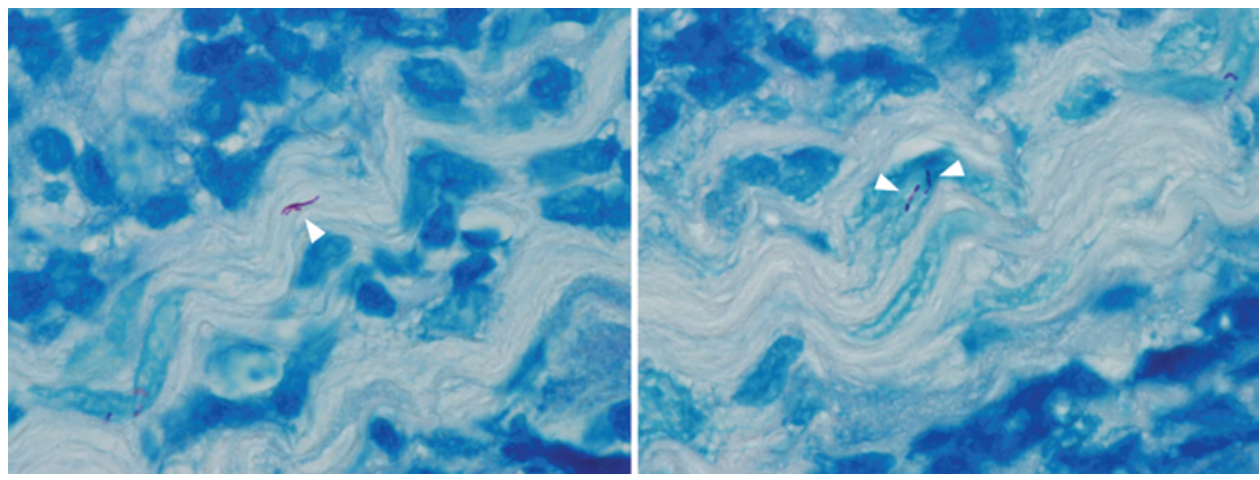

FIG. 4. Nerve biopsy showing several acid-fast organisms staining purple (arrowheads). Fite acid-fast stain, original magnification $\times 1000$. Figure is available in color online only.

respond with successful treatment, so that a lack of enhancement does not preclude the diagnosis of leprosy. ${ }^{18}$ Ultrasound has also been used to evaluate lepromatous nerves and may demonstrate diffuse thickening as well as endoneural color flow signals that correspond to symptomatic neural involvement and may resolve with successful treatment. ${ }^{18}$ Computerized tomography may also demonstrate nerve thickening as well as calcifications. ${ }^{29}$ Some cases of leprosy will present with an intraneural abscess and may be mistaken for an isolated nerve sheath tumor or sarcoma. ${ }^{6,11}$

\section{Fascicular Biopsy}

Given the patient's travel history, a clinical presentation suggestive of multiple nerve involvement, and the lack of clear cutaneous manifestation, we believed that surgical exploration and biopsy were warranted. Fascicular biopsy is thought to be contraindicated in the diagnosis of leprosy given the risk of damaging the parent nerve. ${ }^{18,28}$ In our experience, fascicular biopsy of some proximal nerves such as the sciatic nerve is well tolerated, and based on the established practice of ulnar nerve fascicular transfer for brachial plexus reconstruction, we believed that the need for tissue diagnosis in this case outweighed the risk especially since the delayed treatment of ulnar nerve lesions is known to lead to permanent atrophy and contractures. ${ }^{15}$

\section{Medical Treatment}

Multidrug therapy and a course of steroids are the primary treatment strategy in patients with leprosy. The treatment varies based on whether the patient is categorized in the paucibacillary or multibacillary end of the disease spectrum and continues for 12 to 24 months, respectively. ${ }^{33}$ Despite adequate treatment, patients may have a progressive decline in neurological function during treatment. Notably, even after $M$. leprae has been killed by antimycobacterial treatment, the dead bacilli and their antigens can remain in tissues for several years, offering continuing stimuli for injury. ${ }^{27}$ The exact cause of this is not clear, but it has been noted that apoptosis in in vitro studies of various cell types can be triggered by the ingestion of dead but not live M. leprae. ${ }^{26}$ Host immune response to infected Schwann cells and perineural fibrosis may also play a role and provide the rationale for using steroids acutely. A delayed decline in neurological function can also occur fol- lowing successful treatment, again for unclear reasons. ${ }^{8,10}$ Overall, recovery primarily depends on the severity and duration of neural deficit prior to treatment, so delaying diagnosis with serial imaging can be detrimental in these cases. For those practicing in the United States, the National Hansen's Disease Clinical Center in Baton Rouge, Louisiana, can be a valuable resource. It offers free consultations, free pathological review of skin biopsies, free antibiotics for leprosy treatment, and free educational materials. The WHO also offers multidrug therapy free of cost to those suffering from leprosy.

\section{Surgical Decompression of Lepromatous Nerves}

Pertinent to neurosurgeons is the fact that decompressive surgery as an adjunctive treatment in leprosy has a long and controversial history. ${ }^{12,21}$ As previously noted, leprosy typically involves major nerves at and above areas associated with compressive neuropathy. Relative compression of the nerves in their fibro-osseous tunnels can be demonstrated by imaging studies and at surgery. ${ }^{18,21,35}$ When explored, the nerves, as in our case, have a dense fibrotic consistency. The fibrotic epineurium and external compression are thought to create relative venous obstruction, capillary stasis, edema, and ischemia. External decompression and epineurotomy have been used in an effort to improve overall outcomes in patients with leprosy. ${ }^{12}$ We decompressed the ulnar nerve at the time of biopsy in our case to alleviate any contribution of compression to his deficit, to prevent potential worsening compression in the face of swelling related to the biopsy, and to theoretically maximize his neural regeneration. ${ }^{14,25}$ We believed that the small added morbidity in this case was justified given the typically poor prognosis seen with severe ulnar palsy at the arm level. ${ }^{3,7,15} \mathrm{Had}$ we been able to make the diagnosis of leprosy in this case without the biopsy, however, we would have recommended initial treatment with multidrug therapy and steroids and would not necessarily have intervened surgically unless his pain had not improved.

Numerous reports suggest various benefits of surgical decompression, including improved neurological status, less deformity, and alleviated pain, ${ }^{12,21}$ but the nature of these studies has called into question the true effectiveness of surgery. The literature relating to this issue has been summarized in a Cochrane review last updated in 
2012. ${ }^{34,35}$ No clear statistical benefit from surgical decompression could be demonstrated based on the existing literature, and recommendations have been made for careful randomized controlled trials. ${ }^{21,35}$ As per Husain and Mishra, ${ }^{12}$ we disagree with this recommendation given that the number of uncontrollable variables precludes an ability to get adequately comparable groups. The variable nature of the host response, occasional spontaneous recovery without treatment, clinical worsening during and even months after successful treatment, variable number of nerves and extent of their involvement, variable ability of individual nerves to recover from injury, inability to blind surgical trials, and inability to obtain meaningful outcome measures in the field setting where most care is given make the likelihood of obtaining a legitimate answer regarding surgical decompression in a randomized multicenter trial essentially nil. Large clinical registries and/ or experimental studies in armadillos may help provide some insight in the future, but for now the decision about decompression will need to be made on a case-by-case basis. In our patient, biopsy was critical in making the diagnosis and initiating definitive medical treatment. As his ulnar nerve function was only moderately improved after 8 months of treatment, it is not clear if our decompression added any benefit.

\section{Conclusions}

In summary, leprosy is a cause of significant neurological morbidity worldwide, and because it involves nerves near regions of entrapment such as the ulnar nerve at the elbow, neurosurgeons may be involved in the care of patients with this disease. Diagnosis may be difficult in nonendemic areas since electrodiagnostic and imaging studies are not specific to leprosy. In cases of pure neuritic leprosy with no significant cutaneous manifestations, diagnosis can be particularly challenging and may necessitate fascicular biopsy. Surgical decompression in individual cases with no response to therapy or with clinical worsening may be appropriate but remains controversial. Although the bacteria can be eradicated with multidrug therapy, a delay in diagnosis can lead to permanent neurological morbidity, so leprosy needs to be considered in the differential diagnosis of neuropathy even in nonendemic areas of the world.

\section{References}

1. Agrawal A, Pandit L, Dalal M, Shetty JP: Neurological manifestations of Hansen's disease and their management. Clin Neurol Neurosurg 107:445-454, 2005

2. Antunes SLG, Chimelli L, Jardim MR, Vital RT, Nery JADC, Corte-Real S, et al: Histopathological examination of nerve samples from pure neural leprosy patients: obtaining maximum information to improve diagnostic efficiency. Mem Inst Oswaldo Cruz 107:246-253, 2012

3. Barbour J, Yee A, Kahn LC, Mackinnon SE: Supercharged end-to-side anterior interosseous to ulnar motor nerve transfer for intrinsic musculature reinnervation. J Hand Surg Am 37:2150-2159, 2012

4. Bathala L, Kumar K, Pathapati R, Jain S, Visser LH: Ulnar neuropathy in Hansen disease: clinical, high-resolution ultrasound and electrophysiologic correlations. J Clin Neurophysiol 29:190-193, 2012
5. Beekman R, Slooff WBM, Van Oosterhout MFM, Lammens M, Van Den Berg LH: Bilateral intraneural perineurioma presenting as ulnar neuropathy at the elbow. Muscle Nerve 30:239-243, 2004

6. Bhushan C: Solitary tuberculoid Hansen lesion of the ulnar nerve. J Neurosurg 93:898, 2000

7. Brown JM, Yee A, Mackinnon SE: Distal median to ulnar nerve transfers to restore ulnar motor and sensory function within the hand: technical nuances. Neurosurgery 65:966978, 2009

8. Cardoso F de M, De Freitas MRG, Escada TM, Nevares MT, Nascimento OJ: Late onset neuropathy in leprosy patients released from treatment: not all due to reactions? Lepr Rev 84:128-135, 2013

9. Dastur DK: Pathology and pathogenesis of predilective sites of nerve damage in leprous neuritis. Nerves in the arm and the face. Neurosurg Rev 6:139-152, 1983

10. Dogra S, Kumaran MS, Narang T, Radotra BD, Kumar B: Clinical characteristics and outcome in multibacillary (MB) leprosy patients treated with 12 months WHO MDT-MBR: a retrospective analysis of 730 patients from a leprosy clinic at a tertiary care hospital of Northern India. Lepr Rev 84:6575,2013

11. Finkelstein S, Sima AA, Lougheed WM, Gentili F, Keystone JS: Pure neural tuberculoid leprosy simulating a peripheral nerve tumor. Neurosurgery 10:771-774, 1982

12. Husain S, Mishra B: Decompression of peripheral nerve trunks in leprosy to prevent the development and progression of deformities. Indian J Orthop 42:78-82, 2008

13. Jardim MR, Antunes SLG, Santos AR, Nascimento OJM, Nery JAC, Sales AM, et al: Criteria for diagnosis of pure neural leprosy. J Neurol 250:806-809, 2003

14. Johnston RB, Zachary L, Dellon AL, Mackinnon SE, Gottlieb L: The effect of a distal site of compression on neural regeneration. J Reconstr Microsurg 9:271-275, 1993

15. Kim DH, Han K, Tiel RL, Murovic JA, Kline DG: Surgical outcomes of 654 ulnar nerve lesions. J Neurosurg 98:9931004, 2003

16. Kumar B, Kaur I, Dogra S, Kumaran MS: Pure neuritic leprosy in India: an appraisal. Int J Lepr Other Mycobact Dis 72:284-290, 2004

17. Lee KH, Moon KS, Yun SJ, Won YH, Lee JH, Lee MC, et al: Brain involvement by leprosy presenting as a frontal cystic lesion. J Neurosurg 121:184-188, 2014

18. Martinoli C, Derchi LE, Bertolotto M, Gandolfo N, Bianchi S, Fiallo P, et al: US and MR imaging of peripheral nerves in leprosy. Skeletal Radiol 29:142-150, 2000

19. Mauermann ML, Amrami KK, Kuntz NL, Spinner RJ, Dyck PJ, Bosch EP, et al: Longitudinal study of intraneural perineurioma-a benign, focal hypertrophic neuropathy of youth. Brain 132:2265-2276, 2009

20. Nacey NC, Almira Suarez MI, Mandell JW, Anderson MW, Gaskin CM: Intraneural perineurioma of the sciatic nerve: an under-recognized nerve neoplasm with characteristic MRI findings. Skeletal Radiol 43:375-379, 2014

21. Nickerson DS, Nickerson DE: A review of therapeutic nerve decompression for neuropathy in Hansen's disease with research suggestions. J Reconstr Microsurg 26:277-284, 2010

22. Ooi WW, Srinivasan J: Leprosy and the peripheral nervous system: basic and clinical aspects. Muscle Nerve 30:393409, 2004

23. Rodriguez G, Pinto R, Gomez Y, Rengifo ML, Estrada OL, Sarmiento M, et al: Pure neuritic leprosy in patients from a high endemic region of Colombia. Lepr Rev 84:41-50, 2013

24. Roux A, Tréguier C, Bruneau B, Marin F, Riffaud L, Violas $\mathrm{P}$, et al: Localized hypertrophic neuropathy of the sciatic nerve in children: MRI findings. Pediatr Radiol 42:952958,2012 
25. Schoeller T, Otto A, Wechselberger G, Pommer B, Papp C: Distal nerve entrapment following nerve repair. Br J Plast Surg 51:227-230, 1998

26. Scollard DM: The biology of nerve injury in leprosy. Lepr $\operatorname{Rev}$ 79:242-253, 2008

27. Scollard DM, Stryjewska B: Epidemiology, microbiology, clinical manifestations, and diagnosis of leprosy. UpToDate. (http://www.uptodate.com/contents/epidemiologymicrobiology-clinical-manifestations-and-diagnosis-ofleprosy) [Accessed April 16, 2015]

28. Sharma R, Lahiri R, Scollard DM, Pena M, Williams DL, Adams LB, et al: The armadillo: a model for the neuropathy of leprosy and potentially other neurodegenerative diseases. Dis Model Mech 6:19-24, 2013

29. Slim FJ, Faber WR, Maas M: The role of radiology in nerve function impairment and its musculoskeletal complications in leprosy. Lepr Rev 80:373-387, 2009

30. Suzuki K, Akama T, Kawashima A, Yoshihara A, Yotsu RR, Ishii N: Current status of leprosy: epidemiology, basic science and clinical perspectives. J Dermatol 39:121-129, 2012

31. Truman RW, Singh P, Sharma R, Busso P, Rougemont J, Paniz-Mondolfi A, et al: Probable zoonotic leprosy in the southern United States. N Engl J Med 364:1626-1633, 2011

32. US Department of Health and Human Services: National Hansen's Disease (Leprosy) Program. (www.hrsa.gov/ hansensdisease) [Accessed April 16, 2015]

33. US Department of Health and Human Services: Recommended treatment regimens. National Hansen's Disease (Leprosy) Program. (www.hrsa.gov/hansensdisease/diagnosis/ recommendedtreatment.html) [Accessed April 16, 2015]

34. Van Veen NHJ, Schreuders TAR, Theuvenet WJ, Agrawal A, Richardus JH: Decompressive surgery for treating nerve damage in leprosy. Cochrane Database Syst Rev 12: CD006983, 2012
35. Van Veen NHJ, Schreuders TA, Theuvenet WJ, Agrawal A, Richardus JH: Decompressive surgery for treating nerve damage in leprosy. A Cochrane review. Lepr Rev 80:3-12, 2009

36. Weiland TL, Scheithauer BW, Rock MG, Sargent JM: Inflammatory pseudotumor of nerve. Am J Surg Pathol 20:12121218,1996

\section{Author Contributions}

Conception and design: Payne, Baccon, Dossett, Scollard, Harbaugh. Acquisition of data: all authors. Analysis and interpretation of data: Scollard, Harbaugh. Drafting the article: Payne, Baccon, Dossett, Scollard, Harbaugh. Critically revising the article: Payne, Baccon, Harbaugh. Reviewed submitted version of manuscript: Payne, Dossett, Scollard, Byler, Patel, Harbaugh. Approved the final version of the manuscript on behalf of all authors: Payne. Study supervision: Harbaugh.

\section{Supplemental Information}

Previous Presentation

Portions of this work were presented in poster form at the 82nd AANS Annual Scientific Meeting held in San Francisco, California, on April 5-9, 2014.

\section{Correspondence}

Russell Payne, Department of Neurosurgery, Penn State Milton S. Hershey Medical Center, 30 Hope Dr., Bldg. B, Ste. 1200, Hershey, PA 17033. email: russell.a.payne@gmail.com. 